\title{
Algorithmic Approach for Designing Plasmonic Nanotweezers
}

\author{
Neuton Li ${ }^{1}$, Jasper Cadusch ${ }^{2}$, And Kenneth Crozier ${ }^{1,2, *}$ \\ ${ }^{1}$ School of Physics, University of Melbourne, Victoria 3010, Australia \\ ${ }^{2}$ Department of Electrical and Electronic Engineering, University of Melbourne, Victoria 3010, Australia \\ *Corresponding author: kcrozier@unimelb.edu.au
}

Received XX Month XXXX; revised XX Month, XXXX; accepted XX Month XXXX; posted XX Month XXXX (Doc. ID XXXXX); published XX Month XXXX

\begin{abstract}
We use machine learning (simulated annealing) to design plasmonic nanoapertures that function as optical nanotweezers. The nanoapertures have irregular shapes that are chosen by our algorithm. We present electromagnetic simulations that show that these produce stronger field enhancements and extraction energies than nanoapertures comprising double nanoholes with the same gap geometry. We show that performance is further improved by etching one or more rings into the gold surrounding the nanoaperture. Lastly, we provide a direct comparison between our design and work that is representative of the state of the art in plasmonic nanotweezers at the time of writing.
\end{abstract}

\section{(C) 2019 Optical Society of America}

OCIS codes: (250.5403) Plasmonics; (080.1753) Computation methods. (350.4855) Optical tweezers or optical manipulation

http://dx.doi.org/10.1364/OL.99.099999

There is currently considerable interest concerning the use of light to exert forces on objects in the technique known as optical tweezers. This is in part motivated by the possibilities it affords for investigating microscopic biological systems, e.g. those incorporating complex fluids ${ }^{1}$. Recent developments ${ }^{2}$ include new trapping configurations and new applications. For the field of nanoscience, the ability to trap very small particles is advantageous. However, in conventional optical tweezers, in which a laser beam is focused with a lens, there is an upper limit on the force that can be exerted on a particle of a given size and refractive index with a given laser power and wavelength. This is because the gradient force is proportional to the gradient of the intensity (e.g. see Ref [3]), and conventional optical tweezers are subject to the diffraction limit. The gradient force is also approximately proportional to particle volume. This makes the trapping of nanoparticles challenging.

Using plasmonic nanostructures, light can be confined to deeply-subwavelength regions. This has motivated their use in trapping, i.e. as plasmonic nanotweezers. Trapping with plasmonic nanoantennas has been demonstrated, but heating has been shown to play an important role 4 . In this regard, it has been shown that plasmonic nanoapertures offer the benefit of reduced heating 5 , as the metal film itself acts as a heat-sink. Plasmonic nanoapertures are also relatively easy to fabricate. Plasmonic nanoapertures have therefore proven effective as nanotweezers, and configurations that have been studied include circular apertures ${ }^{6}$, coaxial apertures ${ }^{7,8}$ and double nanoholes (DNH) ${ }^{9,10}$. Thus far, however, these have been generally designed by first using human intuition to decide upon the basic structure and then performing electromagnetic simulations to fine tune it by varying some aspect of its geometry. This leads us to consider whether an algorithmic approach might be superior. This is the topic of this Letter. We are motivated by recent demonstrations of the efficacy of algorithmic approaches in photonics, e.g. in topology optimization ${ }^{11}$ coupled with inverse electromagnetic design ${ }^{12}$. In this work, we use a metaheuristic optimization algorithm (simulated annealing) to design a nanoaperture in a gold film, with the aim of maximizing a figure of merit (FOM) that is the electric field enhancement at the center of the nanoaperture. We show that this produces a nanoaperture design that has an unusual shape, with improved performance over existing structures.

Our optimization algorithm takes its initial structure to be a DNH aperture designed for operation at a wavelength of $1064 \mathrm{~nm}$. To enable a fair comparison between the original DNH and our optimized design, we maintain the curvature around the gap region, i.e. the positions of the green dots of Fig. 1a are fixed. Our algorithm can be summarized as follows. In each iteration step, we select one non-fixed point along the edge of the nanoaperture (blue dots of Fig. 1a) and shift it to a new position sampled from a random uniform distribution within the vicinity of the original point. The distance that the point shifts has an upper bound (initially $20 \mathrm{~nm}$ ) that gradually reduces as the algorithm progresses. Then the electric field distribution is simulated (COMSOL Multiphysics) and the value of the FOM is determined. The algorithm then compares the FOM of the new design to that of the previous design. If the FOM is superior to the previous FOM, then the current design is accepted. If the FOM is inferior, then there is a small but non-zero probability that it is still accepted $^{13}$. The probability function can be expressed as

$$
\operatorname{Pr}=\frac{1}{1+\exp \left(\frac{\text { FOM }_{\text {old }}-F O M_{\text {new }}}{\mathrm{T}}\right)} \text {. }
$$

From Eqn (1), it can be seen that this probability increases as the difference between the old and new FOMs becomes smaller. The 
temperature $T$ starts with an initial value of 100 and decreases exponentially with each iteration step. Hence as the algorithm progresses, the probability of accepting an inferior design decreases. Another feature that is implemented is the 're-annealing' of the system, that is resetting the $T$ parameter. This allows the algorithm to accept a much worse design in the hopes of leaving any local maxima in which it may find itself. In this work, the $T$ parameter is reset every 130 iterations.
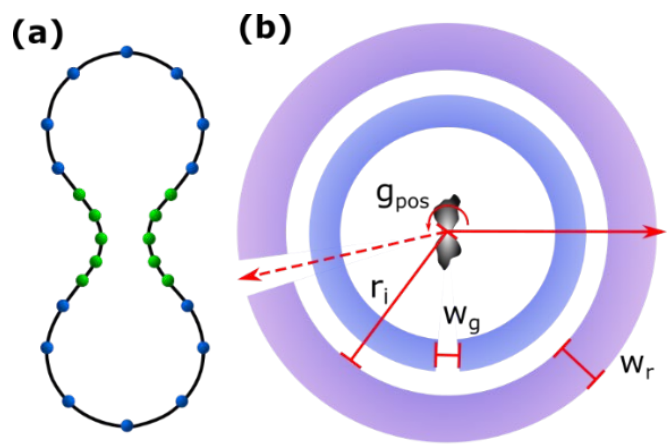

(c)

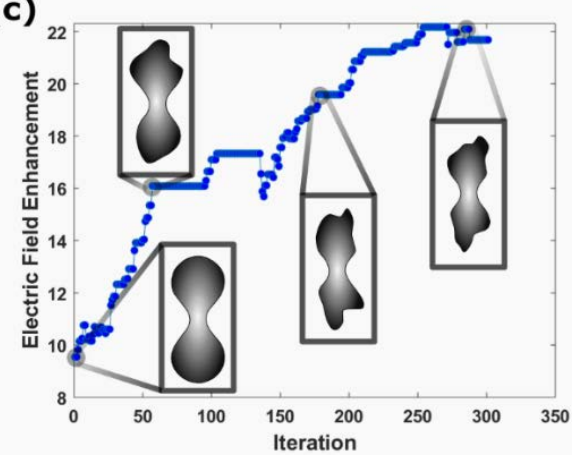

Figure 1. (a) Schematic of nanoaperture, showing points through which an interpolation curve generates the outline of the shape. Gold is removed from interior. (b) Schematic of nanoaperture with concentric rings. (c) Field enhancement vs iteration step.

The nanoaperture simulated is formed in a gold film (100 nm thick) on a glass substrate. The medium above the gold (and within the nanoaperture) is water. Illumination is from the water side and is an $x$-polarized (i.e. across the nanoaperture gap) plane wave at normal incidence with $\lambda=1064 \mathrm{~nm}$. The FOM that the algorithm aims to maximise is the electric field enhancement (EFE) at the very center of the nanoaperture and halfway through the film, defined to be $E F E=\frac{E_{t o t}}{E_{\text {inc }}}$, where $E_{\text {tot }}$ and $E_{\text {inc }}$ are the norms of the total and incident fields, respectively.

We first consider the performance predicted for the nanoaperture that results from this optimization. Later, we consider the effect of adding rings (Fig. 1b). The results of an iteration sequence for the nanoaperture-only case are shown as Fig. 1 c. We stop at 300 iterations, at which the field enhancement is in excess of double that of the starting design. To further illustrate the progress of the algorithm, a few selected nanoaperture shapes are included in Fig. 1c. The nanoaperture that achieves the highest FOM is that corresponding to iteration step 274. This is the optimal design produced by our algorithm for this gap width and is studied in the remainder of this Letter. To compare it to the original DNH, we present the electric field distribution of both structures as Fig. 2a, b. These are plotted halfway through the gold film. As expected, the region with the highest EFE is in the narrow gap region at the center. It can be seen that our shaped aperture (SA) has a maximum EFE that is in excess of double that of the original DNH structure.
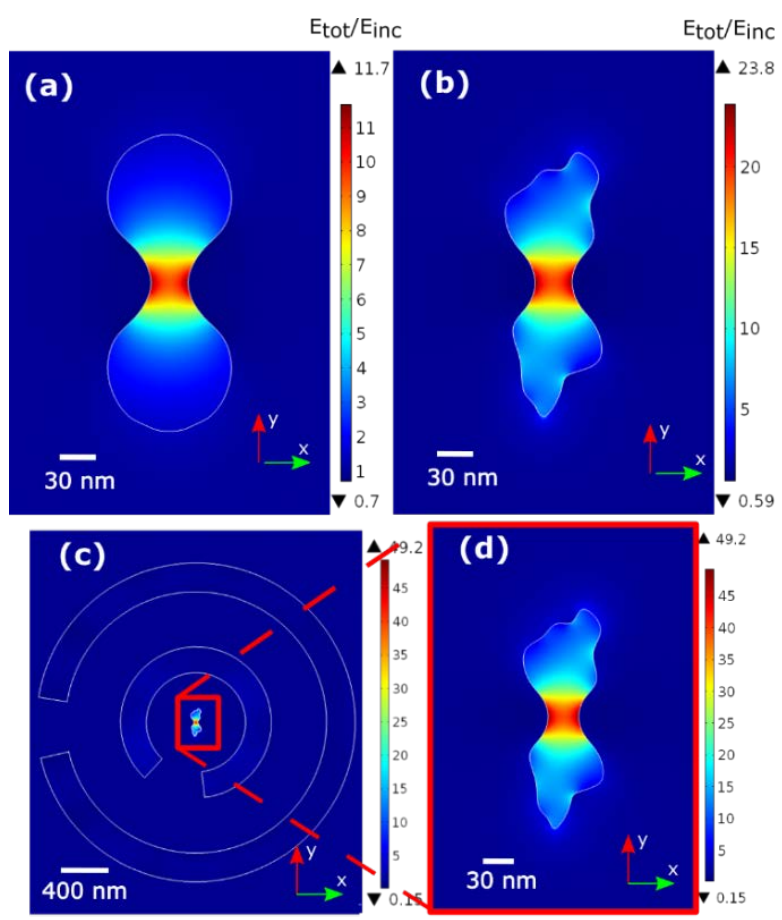

Figure 2. Electric field enhancements for designs with $30 \mathrm{~nm}$ gaps, (a) Original DNH, (b) Optimized structure, (c) Optimized structure with rings. (d) Zoom-in of optimized structure with rings.

We next allow the algorithm to include one or more open rings (i.e. annuli for which the gold is removed, Fig. 1b) into the existing designed structure. This is motivated by the possibility that such rings might increase the coupling of incident light into the central region where the nanoaperture is positioned. Each ring is specified by its inner radius $r_{i}$, thickness $w_{r}$ (width), gap width $w_{g}$ and gap position $g_{p o s}$. Each of these parameters can change independently of any other ring. A gap (i.e. where gold is not removed) is left in each ring to ensure continuity of the gold films, thereby allowing it to be effective at dissipating heat generated by optical absorption. The outermost ring radius is constrained to be less than $1300 \mathrm{~nm}$ to ensure that the simulation time is tractable. The nanoaperture shape is not modified, i.e. the shape of Fig. $2 \mathrm{~b}$ is used. The result of our optimization is a design containing two rings (Fig. 2c, d), that produces a peak EFE that is in excess of quadruple that of the original DNH. The thicknesses are $w_{r}=202 \mathrm{~nm}$ and $w_{r}=$ $225 \mathrm{~nm}$ for the inner and outer rings respectively. The distance from the inner ring to the outer ring is $430 \mathrm{~nm}$. For comparison, we also simulate the case of adding the two rings to the original DNH. Due to space constraints we do not include figures of these results. We find that the peak EFE is $\sim 1.6$ times that of the original DNH.

We next quantify the trapping performance that might be expected. To do so, we calculate the forces experienced by a spherical test particle (diameter $10 \mathrm{~nm}$, refractive index =1.6). The forces are determined by calculating the Maxwell stress tensor (MST) at various points within the domain, for plane wave 
illumination at normal incidence ( $x$-polarized, $I_{0}=6.67 \mathrm{~mW} /$ $\mu m^{2}$ ). Cubic spline interpolation is then performed over these points and the extraction energy is determined by calculating the line integral over the force field ${ }^{14}$. This quantity is often referred to as the "trapping potential" (e.g. [7]) but we note that this is only strictly true if the force field is solely conservative ${ }^{15}$. Nonetheless, we anticipate that this approach is accurate enough for the purpose of predicting the relative performance of the various designs we consider. In Fig. 3a, the force is calculated along the $y$-axis halfway through the gold film of the aperture. The $y$ component of the force is by far the most significant in terms of magnitude. In each case $F_{y}$ is positive for negative $y$, and negative for positive $y$, thus restoring the particle's position to the center. It can be seen that the SA (Fig. $3 c$ ) produces a extraction energy that is deeper than that of the original DNH (Fig. 2b) by a factor of $\sim 4.5$. Furthermore, when the rings are added, the resultant design (Fig. 2c, d) produces an extraction energy that is $\sim 19$ times deeper than the original DNH. The maximum extraction energy that is possible has depth $\sim 1.2 \mathrm{kB}$ for a $10 \mathrm{~nm}$ particle at the center of the gap. In Fig. 3b, the force is calculated along the $z$-axis normal to the substrate surface and in the middle of the gap. In this case, the most significant component of the force is in the $z$ direction. Noting that the gold film comprises the region between $z=0 \mathrm{~nm}$ to $z=100 \mathrm{~nm}$, we see that the force acting on the particle rises steeply comes to within several tens of nanometers of the aperture. The greatest force is experienced near the cusp of the aperture and in the negative $z$ direction. This acts to pull the particle further into the nanoaperture. The extraction energy plots of Fig. 3d show that, along this direction, the optimal design again represents an improvement over the original DNH. The shape of the well suggests that the particle will be trapped within the nanoaperture, below the gold film surface.

We next apply our algorithm to a DNH structure with a gap of $10 \mathrm{~nm}$, to explore what might be possible with a structure that is expected to be achievable by advanced nanofabrication. The shape of the initial DNH is determined by sweeping the geometric parameters of the structure, i.e. the hole radii and their separation, and choosing the design with the highest EFE (Fig. 4a). The algorithm then proceeds in the same way as before and the optimal structure that it arrives at again has an unusual shape (Fig. 4b). It does not share the same form as the previous structure with the larger gap (Fig. 2b). The maximum field enhancement can be seen to be increased by a factor of 1.6 times through the optimization process. We next include rings with the same geometry as before, as we expect these to offer the same benefits for this SA (with gap 10 $\mathrm{nm}$ ) as for the SA (with gap $30 \mathrm{~nm}$ ) considered earlier. From Fig. 4c, it can be seen that the rings results in an EFE (of 106) that is approximately triple that of the starting structure (with EFE 35.5).

To predict the trapping performance of the nanoaperture with the smaller gap and the rings (Fig. 4c-d), we again calculate the force on the particle as a function of position and integrate to find the extraction energy. We again use plane wave illumination (polarized across the gap), with $I_{0}=6.67 \mathrm{~mW} / \mu \mathrm{m}^{2}$. Our spherical test particle (refractive index 1.6) is taken as having a diameter of $9 \mathrm{~nm}$ to ensure that it is slightly narrower than the nanoaperture gap. The results (not shown here due to page limits) reveal that the extraction energy (for integration along z-direction) reaches $\sim 6 \mathrm{k}_{\mathrm{B}} \mathrm{T}$, i.e. around five times greater than of the previous structure (Fig 3c-d). We anticipate that it would be interesting to investigate the fabrication of such a nanoaperture using advanced nanofabrication methods ${ }^{16}$.
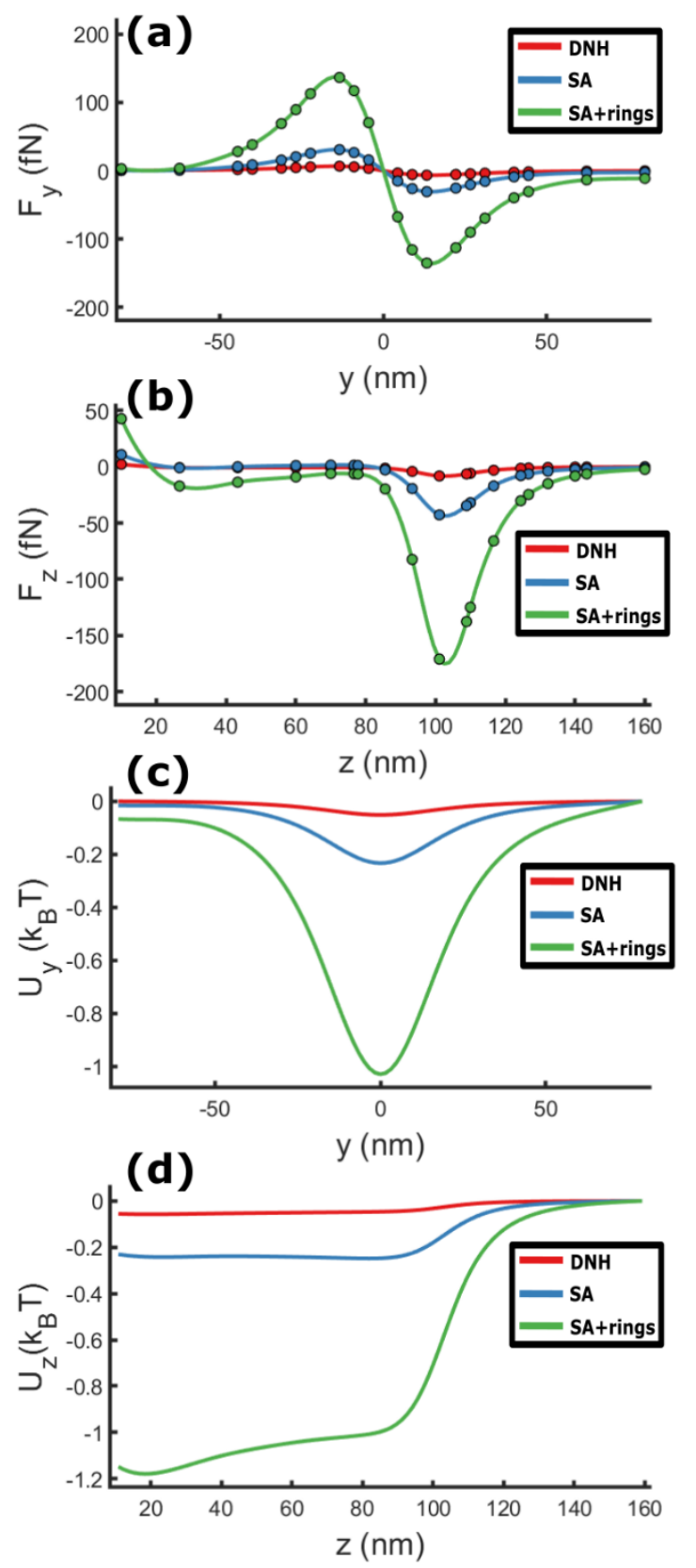

Figure 3. Simulations of double nanohole (DNH), shaped aperture without ring (SA), and shaped aperture with rings (SA+rings). (a) Force (y-component) vs $y$-position with $x=0 \mathrm{~nm}$ and $z=50 \mathrm{~nm}$. (b) Force (zcomponent) vs $z$-position with $x=0 \mathrm{~nm}$ and $y=0 \mathrm{~nm}$. Surface of glass substrate corresponds to $z=0 \mathrm{~nm}$. (c) Extraction energy vs $y$ position. (d) Extraction energy vs z-position. 

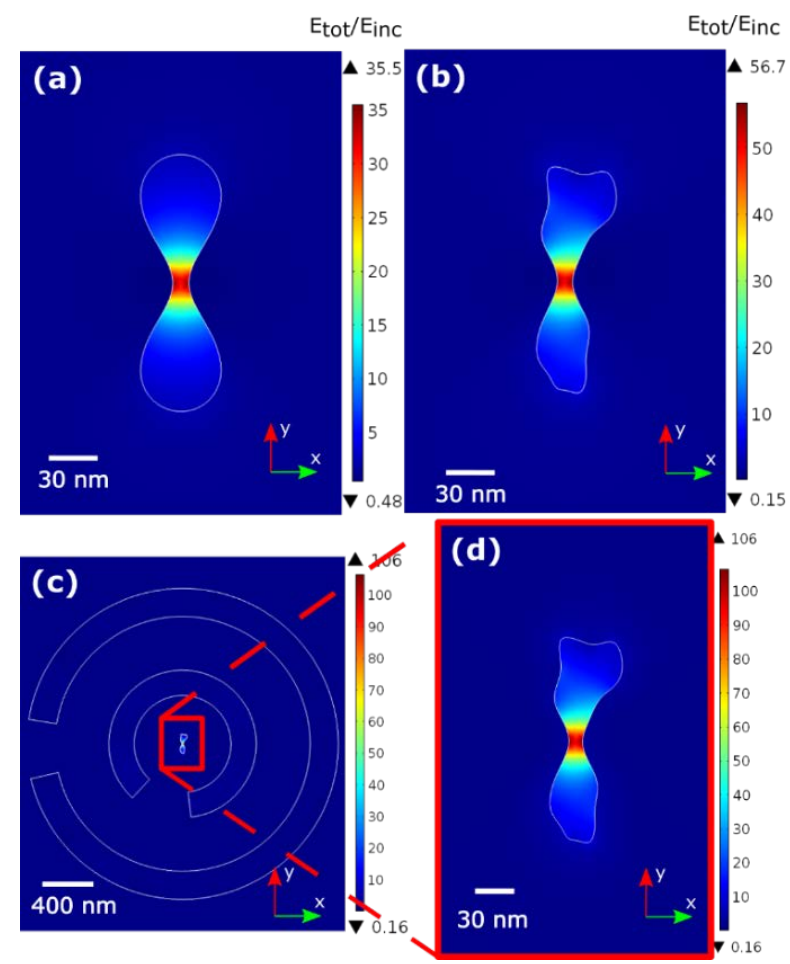

Figure 4. Electric field enhancements for design with $10 \mathrm{~nm}$ gap, (a) Original DNH, (b) Optimized structure without rings. (c) Optimized structure, with rings. (d) Zoom in view to center of panel c.

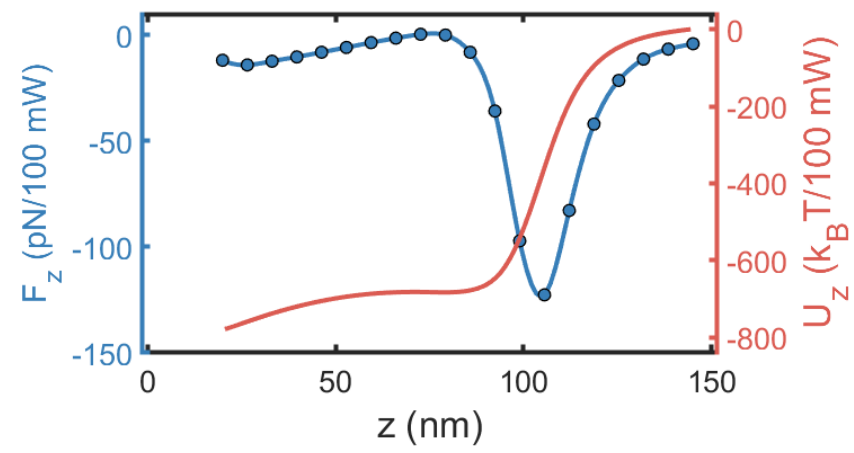

Figure 5. Force and extraction energy for spherical particle (diameter $29 \mathrm{~nm}, \mathrm{n}=1.6$ ) as trapped by structure of Fig. 2c-d. (Left) Force vs $z$-position. (Right) Corresponding extraction energy vs $z-$ position.

We next compare our design with one that represents the state-of-the-art at the time of writing. For the latter, we consider Yoo et al [8], who investigate a coaxial nanoaperture with a $10 \mathrm{~nm}$ gap. In Ref [8], it is shown that this yields an extraction energy (termed "trapping potential") of $\sim 30 \mathrm{k}_{\mathrm{B}} \mathrm{T}$ for a nanosphere ( $30 \mathrm{~nm}$ diameter, $\mathrm{n}=1.6$ ) with a focused beam (power $100 \mathrm{~mW}$, numerical aperture $\mathrm{NA}=1.25)$. For comparison, we again consider our optimal structure of Fig. 2c-d, but this time with a focused beam (100 mW, NA=1.25). We take our nanosphere to have diameter $29 \mathrm{~nm}$ and $\mathrm{n}=1.6$. We determine the MST and again integrate to find the extraction energy. The results are shown in Fig. 5. As the particle is larger than before (Fig. 3), it has a larger polarizability ${ }^{17}$. The extraction energy has a depth of $\sim 800 \mathrm{k}_{\mathrm{B}} \mathrm{T}$, i.e. more than an order of magnitude deeper than that of Ref [8]. This is even though our structure (Fig. $2 \mathrm{c}-\mathrm{d}$ ) has a wider gap (of $30 \mathrm{~nm}$ ) than that of Ref [8] (10 nm gap).
In summary, we demonstrate that machine learning enables plasmonic nanotweezers to be designed that surpass those produced by traditional approaches

Funding. Australian Research Council (DP180104141 and FT140100577)

Acknowledgment. The authors thank Dr Vivek Raj Shrestha and Dr Zhe Xu for helpful discussions.

Disclosures. The authors declare no conflicts of interest.

\section{References}

1. S. Zhang, L. J. Gibson, A. B. Stilgoe, I. A. Favre-Bulle, T. A. Nieminen, and H. Rubinsztein-Dunlop, Optica 4, 1103 (2017).

2. D. Gao, W. Ding, M. Nieto-Vesperinas, X. Ding, M. Rahman, T. Zhang, C. Lim, and C.-W. Qiu, Light Sci. Appl. 6, e17039 (2017).

3. P.C. Chaumet and M. Nieto-Vesperinas, Opt. Lett. 25, 1065 (2000).

4. S. Jones, D. Andrén, P. Karpinski, and M. Käll, M. ACS Photon. 5, 2878 (2018).

5. Q. Jiang, B. Rogez, J.-B. Claude, G. Baffou, and J. Wenger, ACS Photon. 6, 1763 (2019).

6. E.-S. Kwak, T.-D. Onuta, D. Amarie, R. Potyrailo, B. Stein, S. C. Jacobson, W. L. Schaich, and B. Dragnea, J. Phys. Chem. B 108, 13607 (2004).

7. A. A. E. Saleh, and J. A. Dionne, Nano Lett. 12, 5581 (2012).

8. D. Yoo, K. L. Gurunatha, H.-K. Choi, D. A. Mohr, C. T. Ertsgaard, R. Gordon, and S.-H. Oh, Nano Lett. 18, 3637 (2018).

9. H. Xu, S. Jones, B.-C Choi, and R. Gordon, Nano Lett. 16, 2639 (2016).

10. Z. Xu, W. Song and K. B. Crozier, ACS Photon. 5, 2850 (2018).

11. T. Phan, D. Sell, E. W. Wang, S. Doshay, K. Edee, J. Yang, and J. A. Fan, Light Sci. Appl. 8, 48 (2019).

12. A. Y. Piggott, J. Lu, K. G. Lagoudakis, J. Petykiewicz, T. M. Babinec, and J. Vučković, Nat. Photonics 9, 374 (2015).

13. S. Kirkpatrick, C. D. Gelatt, and M.P. Vecchi, Science 220, 671 (1983).

14. M. Ploschner, M. Mazilu, T. F. Krauss, and K. Dholakia, J. Nanophoton. 4, $041570(2010)$

15. M. A. Zaman, P. Padhy, and L. Hesselink, Sci. Rep. 9, 649 (2019).

16. H. Kollmann, X. Piao, M. Esmann, S. F. Becker, D. Hou, C. Huynh, L.-O. Kautschor, G. Bösker, H. Vieker, A. Beyer, A. Gölzhäuser, N. Park, R. Vogelgesang, M. Silies, and C. Lienau, Nano Lett. 14, 4778 (2014).

17. S. E. S. Spesyvtseva, and K. Dholakia, ACS Photon. 3, 719 (2016). 
1. Zhang, S. et al. Ultrasensitive rotating photonic probes for complex biological systems. Optica 4, 1103-1108 (2017).

2. Gao, D. et al. Optical manipulation from the microscale to the nanoscale: fundamentals, advances and prospects. Light: Science \&Amp; Applications 6, e17039- (2017).

3. Chaumet, P. C. \& Nieto-Vesperinas, M. Time-averaged total force on a dipolar sphere in an electromagnetic field. Opt. Lett. 25, 1065-1067 (2000).

4. Jones, S., Andrén, D., Karpinski, P. \& Käll, M. Photothermal Heating of Plasmonic Nanoantennas: Influence on Trapped Particle Dynamics and Colloid Distribution. ACS Photonics 5, 2878-2887 (2018).

5. Jiang, Q., Rogez, B., Claude, J.-B., Baffou, G. \& Wenger, J. Temperature Measurement in Plasmonic Nanoapertures Used for Optical Trapping. ACS Photonics 6, 1763-1773 (2019).

Kwak, E.-S. et al. Optical Trapping with Integrated Near-Field Apertures. The Journal of Physical Chemistry B 108, 1360713612 (2004).

7. Saleh, A. A. E. \& Dionne, J. A. Toward Efficient Optical Trapping of Sub-10-nm Particles with Coaxial Plasmonic Apertures. Nano Letters 12, 5581-5586 (2012).

8. Yoo, D. et al. Low-Power Optical Trapping of Nanoparticles and Proteins with Resonant Coaxial Nanoaperture Using 10 nm Gap. Nano Letters 18, 3637-3642 (2018).

9. $\mathrm{Xu}, \mathrm{H}$. , Jones, S., Choi, B.-C. \& Gordon, R. Characterization of Individual Magnetic Nanoparticles in Solution by Double Nanohole Optical Tweezers. Nano Letters 16, 2639-2643 (2016).

10. Xu, Z., Song, W. \& Crozier, K. B. Direct Particle Tracking Observation and Brownian Dynamics Simulations of a Single Nanoparticle Optically Trapped by a Plasmonic Nanoaperture. ACS Photonics 5, 2850-2859 (2018).

11. Phan, T. et al. High-efficiency, large-area, topology-optimized metasurfaces. Light: Science \& Applications 8, 48 (2019).

12. Piggott, A. Y. et al. Inverse design and demonstration of a compact and broadband on-chip wavelength demultiplexer. Nature Photonics 9, 374 (2015).

13. Kirkpatrick, S., Gelatt, C. D. \& Vecchi, M. P. Optimization by Simulated Annealing. Science 220, 671-680 (1983).

14. Ploschner, M., Mazilu, M., Krauss, T. F. \& Dholakia, K. Optical forces near a nanoantenna. Journal of Nanophotonics 4, 1-13

15. Zaman, M. A., Padhy, P. \& Hesselink, L. Near-field optical trapping in a non-conservative force field. Scientific Reports $\mathbf{9}$, 649 (2019).

16. Kollmann, H. et al. Toward Plasmonics with Nanometer Precision: Nonlinear Optics of Helium-Ion Milled Gold Nanoantennas. Nano Letters 14, 4778-4784 (2014).

17. Spesyvtseva, S. E. S. \& Dholakia, K. Trapping in a Material World. ACS Photonics 3, 719-736 (2016). 